\title{
Resilience Assessment of Urban Emergency Management for Emergencies
}

\author{
Wang $\mathrm{Xu}^{1, \mathrm{a}^{*}}, \mathrm{Wu}$ Tianyan ${ }^{2, \mathrm{~b}}$ \\ ${ }^{1}$ Master of Safety Engineering, Shanghai Institute of Technology \\ ${ }^{2}$ Professor of Engineering Management, Shanghai Institute of Technology
}

\begin{abstract}
In recent years, urban emergency management and urban resilience have gradually become the main focus of research on the sustainable development of cities in the future due to the huge impact brought by the outbreak of epidemics and urban emergencies. Based on the Pressure-State-Response (PSR) model, this study attempts to longitudinal evaluate the time series of Xuhui District in Shanghai from the pressure layer, state layer and response layer. Based on the understanding of the current situation of the city and the change trend of the past years in advance, and combining with the urban evaluation system, a scientific conclusion is drawn that the resilience of Xuhui District of Shanghai is declining year by year, which provides a scientific basis for the decision-making of urban future development and urban resource allocation.
\end{abstract}

\section{Introduction}

In recent years, the rapid development of cities is often accompanied by all kinds of risks and disasters. The excessive occupation of ecological resources by human beings has seriously polluted the environment, and the vulnerability of the urban system has become increasingly prominent.Due to the continuous increase of emergencies faced by human society and the basic nature of urban vulnerability, cities are subjected to more and more negative impacts [1].

The United States and Japan have set an example for the rest of the world with remarkable results in emergency management assessments.Published articles show that scholars prefer to adopt qualitative research methods. For example, Eisenman believes that the United States must seriously reflect on the lessons of the "9.11" incident caused by terrorism, popularize emergency knowledge for all American citizens and enhance their emergency awareness[2].The research of Chinese scholars on the evaluation system of emergency management capability focuses more on the single disaster.Chen Sheng took the 2008 Sichuan earthquake as an example, and from the standpoint of the majority of the affected groups, constructed an index system that could reflect the management ability under the crisis state [3].

Most of the existing researches in China lack discussion on evaluation model and empirical analysis. Although some research ideas have been put forward in the index construction, some index systems are too subjective and lack of theoretical analysis support.Therefore, based on the relatively mature PSR model and using its core logic and framework for reference, this paper further reflects the different characteristics of resilience and related influencing factors.

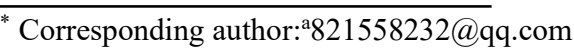

bwty@sit.edu.cn
}

Meanwhile, by means of theoretical and practical research methods, a resilience assessment model for urban emergency management is established on this basis. The toughness level of Xuhui District in Shanghai was investigated and analyzed empirically, and the corresponding toughness improvement strategies were put forward.It not only assessed the advantages and disadvantages of resilience, but also deeply explored the objective laws of urban resilience development on this basis, and carried out in-depth and objective quantitative assessment of urban resilience from the perspective of improving urban emergency management ability.From the theoretical dimension, it can provide a new perspective for the related research on urban resilience, and further broaden the theoretical field of urban resilience research in China, so as to provide better practical guidance.

\section{Materials and Methods}

\subsection{The Study Area}

As an international metropolis and national economic and cultural center, Shanghai faces more emergencies than other cities in China. Meanwhile, due to its basic urban characteristics, such as high building density and population density, special geographical location at the mouth of the Yangtze River, strong mobility of permanent residents, etc., The impact of emergencies on urban development has the characteristics of big impact, long time and complex management mode. 


\subsection{Data Source}

The data used in this paper are from the questionnaire survey conducted by experts, the annual statistical yearbook of Shanghai municipal governments and the public data of government security-related departments from 2015 to 2019.In order to avoid the possibility of contingency in the data obtained from the questionnaire survey, the author invited experts in the fields of urban planning, security and architecture to fill in the questionnaire. However, there may still be problems such as relatively concentrated professional fields and insufficient sample size.

\subsection{Toughness Assessment Method}

\subsubsection{Analytic Hierarchy Process}

Principle of analytic hierarchy process (ahp) is a relatively complex problems, according to the subordinate relations, decomposed into target layer, criterion layer, a multi-level analysis such as the layer structure model, through comparing various elements of the same level, determine the relative importance of each factor in the hierarchy, thus the problem boils down to the bottom to the top is relatively important determining weights.

\subsubsection{Entropy Value Method}

Information entropy can be used to calculate the weight of each index in the evaluation of urban resilience, which can provide objective basis for the comprehensive evaluation system.Build a matrix of $\mathrm{j}($ year/city)* I (indicator).The value of the I index in the JTH year (city) is the value $F_{i j}$ after standardized treatment.

$$
\begin{aligned}
& \mathrm{P}_{i j}=\frac{F_{i j}}{\sum_{j=1}^{M} F_{i j}} \\
& e_{i}=-k \sum_{j=1}^{m} P_{i j} \ln \left(P_{i j}\right) \\
& \mathrm{W}_{\mathrm{i}}=\frac{1-\mathrm{e}_{\mathrm{i}}}{\mathrm{n}-\sum_{\mathrm{i}=1}^{\mathrm{n}} \mathrm{e}_{\mathrm{i}}}
\end{aligned}
$$

$P_{i j}$ is the proportion of each index, $e_{i}$ is the entropy value of the ith index, and $\mathrm{w}_{\mathrm{i}}$ is the entropy weight of the ith index.

\subsubsection{Combined weight optimization}

The index weights of different levels obtained by the subjective weighting method and the objective weighting method are combined and optimized to make comprehensive use of the advantages of the subjective and objective weighting method to the greatest extent.

$$
\mathrm{W}_{\mathrm{i}}=\frac{\mathrm{W}_{1 \mathrm{i}} \mathbf{W}_{2 \mathrm{i}}}{\sum_{\mathrm{i}=1}^{\mathrm{n}} \mathrm{W}_{1 \mathrm{i}} \mathbf{W}_{2 \mathrm{i}}}
$$

$\mathrm{W}_{\mathrm{i}}$ is the combined weight of each index in the final evaluation system, $\mathrm{n}$ is the number of indicators in the evaluation system, $\mathrm{W}_{1 \mathrm{i}}$ is the subjective assigned weight, and $\mathrm{W}_{2 \mathrm{i}}$ is the objective assigned weight.

\subsubsection{Evaluation index method based on PSR model}

Toughness based on PSR model of city emergency management mechanism of action of the figure shows that stress state of toughness and tenacity to improve improve finally promotes the city emergency management response toughness, and the face of emergency for the city's emergency management ductile response layer and reduce the impact on the city, will eventually back to the city emergency management stress state of toughness and tenacity,forming a dynamic and interactive self-adaptive cycle emergency management mechanism.

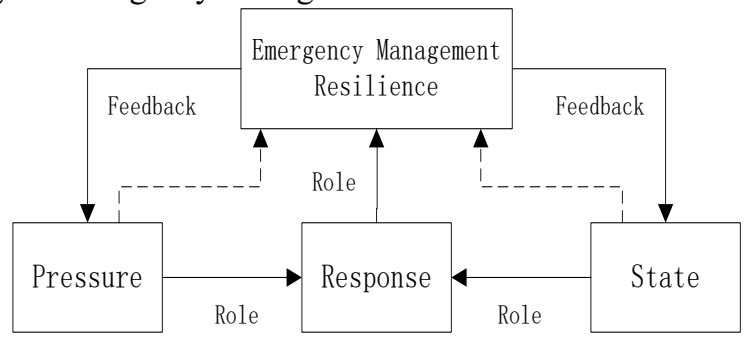

Fig. 1. The mechanism of PSR model

$$
\begin{aligned}
& \mathrm{A}=\sum_{\mathrm{i}=\mathrm{a}_{1}}^{\mathrm{a}_{\mathrm{n}}}\left(\mathrm{Q}_{\mathrm{i}} \times \mathrm{W}_{\mathrm{i}}\right) ; \\
& \mathrm{B}=\sum_{\mathrm{i}=\mathrm{b}_{1}}^{\mathrm{b}_{\mathrm{n}}}\left(\mathrm{Q}_{\mathrm{i}} \times \mathrm{W}_{\mathrm{i}}\right) ; \\
& \mathrm{C}=\sum_{\mathrm{i}=\mathrm{c}_{1}}^{\mathrm{c}_{\mathrm{n}}}\left(\mathrm{Q}_{\mathrm{i}} \times \mathrm{W}_{\mathrm{i}}\right) ; \\
& \mathbf{R}=\frac{\mathbf{C}}{(\mathbf{A}+\mathbf{B})}
\end{aligned}
$$

A is pressure toughness, $\mathrm{B}$ is state toughness, $\mathrm{C}$ is response toughness, $a_{n}$ is the number of pressure layer indicators; $b_{n}$ is the number of indicators in the state layer; $c_{n}$ is the number of indicators in the response layer; $Q_{i}$ is the scoring value of each indicator; $\mathrm{R}$ is the resilience index of urban emergency management for emergencies.

\section{Results \& Discussion}

\subsection{Index Weight}

According to the index weight combination optimization method and formula, the subjective and objective index weights obtained by the analytic hierarchy process and the entropy method respectively, the composite composite index weights of the urban emergency management 
resilience evaluation criteria in Xuhui District of Shanghai were calculated. The results are shown in Table 1:

Table1 Optimized index weight

\begin{tabular}{cccc}
\hline Target Layer & Elements Layer & Index Layer & Weight \\
\hline & External Potential & Number of Emergencies & 0.0458 \\
& Risks & Number of Disaster Accidents & 0.0475 \\
& & Number of Traffic Accidents & 0.1047 \\
Pressure Layer & Community Transportation & 0.0458 \\
& Internal Potential Risk & Construction & 0.0082 \\
& & Household Unemployment Rate & 0.0065 \\
& Convenience Facilities & 0.0491 \\
Characteristics of Urban & Construction & 0.0311 \\
Residents & Proportion of Education & 0.0524 \\
& Expenditure & 0.0491 \\
& Characteristics of Urban & Percentage of Elderly and Children & 0.0344 \\
& Environment & Construction of Living Facilities & 0.0065 \\
& Construction of Medical Resources & 0.0115 \\
& Urban Greening Rate & Number of Disaster & 0.0638 \\
& Emergency Organization & Prevention Drills & 0.0360 \\
& & Emergency Supplies Budget & 0.2684 \\
& & Emergency Plan Construction & \\
& & Information System & 0.1064 \\
& & Construction & 0.0327 \\
& Adaptive Resilience & City GDP &
\end{tabular}

\subsection{Longitudinal Assessment of Resilience in Uemergency Management}

The resilience development of Xuhui District of Shanghai was analyzed based on the annual statistical yearbook of each district government of Shanghai and the public data of government safety related departments from 2015 to 2019, and the longitudinal change of resilience in emergency management was explored.

Based on PSR model to construct the comprehensive evaluation index method and formula, using the above the xuhui district in Shanghai city emergency management comprehensive portfolio weights are toughness evaluation standard, calculated from 2015 to 2019 of xuhui district, Shanghai city emergency management toughness and DengZi pressure, state and response factors of resilience level, such as the process and the results are shown in Table 2:

Table 2. Evaluation Score

\begin{tabular}{ccccc}
\hline & $\begin{array}{c}\text { Pressure } \\
\text { toughness }\end{array}$ & $\begin{array}{c}\text { State } \\
\text { toughness }\end{array}$ & $\begin{array}{c}\text { response } \\
\text { toughness }\end{array}$ & $\begin{array}{c}\text { City } \\
\text { toughness }\end{array}$ \\
\hline 19 & 0.5787 & 0.4117 & 0.6683 & 1.6587 \\
18 & 0.4088 & 0.3804 & 0.6979 & 1.4872 \\
17 & 0.4002 & 0.3889 & 0.7580 & 1.5472 \\
16 & 0.4356 & 0.3746 & 1.5421 & 2.3522 \\
15 & 0.7273 & 0.6697 & 1.5220 & 2.9190 \\
\hline
\end{tabular}

Combined with the urban security situation, the scores of resilience index were divided into five $\mathrm{R}$-value ranges.Under the definition of urban emergency management resilience, the five regions are successively high resilience, medium high resilience, medium low resilience, and low resilience. The toughness level of each year is shown in Table 3:

Table 3. Resilience Rating

\begin{tabular}{ccc}
\hline Level & Toughness Level Interval & Year \\
\hline High & $2.4000 \sim 3.0000$ & 2015 \\
Toughness & & \\
Medium-high & $1.8000 \sim 2.4000$ & 2016 \\
Toughness & & 2017 \\
Medium & $1.2000 \sim 1.8000$ & 2018 \\
Toughness & & 2019 \\
Medium-low & $0.6000 \sim 1.2000$ & \\
Toughness & $0.000 \sim 0.6000$ & \\
Low Toughness & \\
\hline
\end{tabular}

Along with the rapid development of the city, the city level of resilience in the face of emergencies emergency management in decline year by year, although still can maintain at a certain level, but the city managers if not for the corresponding to the direction of the city, and take necessary corrective policy solutions, toughness of city emergency management level have to fall further until the middle and lower toughness even low toughness level of risk.

It reflects that the stress resilience of Xuhui District of Shanghai has a certain correlation with the resilience of urban emergency management. The stress resilience is in the same declining trend, and there is a risk of falling to a lower level if no measures are taken.

This also shows that in recent years, Xuhui District of Shanghai is still greatly affected by the pressure of external and internal potential risks, and the level of social 
emergency management in the urban space is greatly negatively affected.

The urban status of Xuhui District of Shanghai is less disturbed by urban development. The urban environmental characteristics and urban residents' characteristics of Xuhui District of Shanghai change gently with a small range over time. In the long run, there is no trend of significant decrease or growth, and it will continue to maintain the healthy and sustainable characteristics.

Shanghai xuhui district response toughness toughness toughness and stress state, the overall trend and trend of xuhui district, Shanghai city emergency resilience level changes is slightly different, more difficult to predict the future development trend, but by the past five years resilience score and total score interval, the city managers will more resources to maintain and promote city emergency organization ability and adapt the resilience.omote city emergency organization ability and adapt the resilience.

Further analysis shows that urban managers should still give priority to improving the resilience of urban response when making future decisions and policies related to urban security.

\section{Conclusions}

In this study, various influencing factors, such as urban pressure layer, urban state layer and urban response layer, which have different influences on urban emergency management are deeply discussed, so as to understand the effect of improving different urban factors on the resilience of urban emergency management.

This paper builds the toughness evaluation index system of city emergency management framework of xuhui district in Shanghai emergency management status data for empirical foundation, not only can let the city manager of urban emergency management toughness, have a clear understanding of the current situation of as well as for future urban policy and provide a scientific basis for the allocation of resources city.

In addition, relying on Shanghai's exemplary role as an international metropolis and as a leading city in China, the evaluation system method and evaluation results can be better applied and used for reference by other cities in China, and can also promote the establishment of common understanding on the topic of urban emergency management and urban resilience in relevant research in China.

\section{References}

1. Zhai Guofang, Cui Gonghao, Xie Yingxia et al.Risk Society and Resilient City[J]. The City Planning, 2015, 39(12):107-112.

2. Eisenman D.P., Wold C., Fielding J., Long A. ,Setodji C. , Hickey S., Gelberg L. Differences in individuallevel terrorism preparedness in Los Angeles County[J]. American Journal of Preventive Medicine, 2006,30 (1): 1-6.
3. Barnett D.J., EverlyG.S., Parker C. L. ,Links Jonathan M. Applying educational gaming to public health workforce emergency preparedness [J]. American Journal of Preventive Medicine, 2005, 28 (4): 390-395.

4. Daniel H. Evaluating Local government emergency management programs what framework should public managers adopt[J].Public Administration Review ,2010, (4): 236-246.

5. Chen S. Study on the evaluation of the government's emergency response capability in the five worst-hit areas of Wenchuan Earthquake[J].Public Administration Review, 2010, (8): 112-131. 